\title{
Beitrag zur Kenntniss der Mycosis fungoides.
}

\author{
Von \\ Dr. Velhagen \\ in Chemnitz.
}

Am 13. IX. 1900 consultirte mich eine den besseren Ständen angehörende Frau, 72 Jahre alt, in Chemnitz, welche von einer ebenso nngewöhnlichen wie interessanten Krankheit befallen war.

Sie gab an, bis zum Frühjahr des laufenden Jahres stets gesund gewesen zu sein, abgesehen von einzelnen immer rasch vorübergegangenen Erkältungskrankheiten und leichteren Unterleibsstörungen, die sie öfter im Anschluss an Entbindungen zu spüren gehabt habe. Sie habe einen gesunden Mann sowie fünf erwachsene Kinder, welche ebenfalls durchaus gesund und kräftig seien und sich einer gesunden Nachkommenschaft zu erfreuen hätten.

Vor ca. einem halben Jahr habe ihr jetziges Leiden begonnen mit einem starken Jucken der Haut über den ganzen Körper, welches trotz aller von ihr angewandten Hausmittel nicht zu beseitigen gewesen sei. Irgend welche Ausschläge schuppender oder nässender Art oder sonst etwas Ungewöhnliches auf der Haut habe sie dabei nicht bemerkt. Ein ca. zwei Monate langer Aufenthalt in einem erzgebirgischen Badeort habe ihr keine wesentliche Erleichterung gebracht. Das Jucken sei seit einigen Wochen jedoch endlich von selbst besser geworden; sie fühle sich jedoch immer noch matt und hinfällig, trotzdem Appetit, Verdauung etc. ganz leidlich seien.

Der Grund, weshalb sie zu mir als dem ersten Arzt, welchen sie seit langer Zeit consultire, käme, sei eine Geschwulst im rechten äusseren Augenwinkel, die Thränen, Lichtscheu und auch Schmerzen verursache. Die Geschwulst habe sich seit ca. zwei Wochen langsam gebildet.

Status praesens: Es handelt sich um eine durchaus gesund aussehende Dame mit gutem Panniculus adiposus. Ihre Hant ist zwar von Furchen durchzogen, hat aber sonst eine dem Alter angemessene Farbe. Irgend welche Naevi oder Pigmentflecken sind nirgends zu sehen. Es fällt jedoch bei genauer Inspection anf, dass die Haut des Stammes sowohl wie des Gesichtes, des Halses, der Oberschenkel und Oberarme - an einzelnen Stellen etwas vorgebuckelt ist. Bei der darauf vorgenommenen Palpation fühlt man mühelos in der Tiefe zahllose Knötchen von Erbsen- bis Haselnussgrösse, welche sowohl vollständig beweglich sind gegen ihre Unterlage als auch gegen die sie bedeckenden, durchaus intact aussehenden Hautpartien. 
Ihre Consistenz scheint unter dem tastenden und drilckenden Finger im Allgemeinen die nicht ganz reifer Pflaumen zu sein.

Besonders zahlreich sitzen die subcutanen Tumoren im Gesicht, wo kaum eine $2 \mathrm{~cm}$ lange Strecke frei ist. Der Hals scheint etwas weniger ergriffen, noch weniger Brust and Rücken, am Oberschenkel und Oberarm schliesslich ist nur alle Handbreit ein Knötchen za fühlen.

Ausserdem zeigen sich Nuchal-, Axillar-, Clavieular- und Cubitaldrüsen vergrössert, meist aber so wenig, dass sie nur bei grösster Aufmerksamkeit gefühlt werden können. Sehr stark im Verhältniss geschwollen scheint dagegen die Lymphdrüse in der rechten Parotis zu sein.

Die Untersuchung der inneren Organe in Brust und Bauchhöhle ergiebt nichts Abnormes. Eine Milzvergrösserung ist durchaus nicht zu constatiren. An den Schleimhäuten der Nase, des Mundes und Rachens ist nichts Auffallendes erkennbar.

Der Urin ist frei von Eiweiss und Zucker. - Temperatur $=37,1$, Puls 72 .

Der Befund am rechten Auge ist folgender: In der Gegend der Thrảnendrüse sitzt ein die Haut des äusseren Lidwinkels besonders oben ziemlich weit vorbuckelnder Tumor, der von prall elastischer Consistenz und beweglich ist sowohl gegen das ihn bedeckende Lid, als auch gegen den knöchernen Orbitalrand, hinter welchem er verschwindet. Die Conjunctiva palpebrarum et sclerae ist normal, diejenige der Uebergangsfalten dagegen macht den Eindruck einer chemotischen Schwellung: Der Bulbus selbst ist normal, S. mit $+1,0 \mathrm{D}=5 / 7$.

Am linken Ange ist keine Veränderung wahrzunehmen; $\mathrm{S}$. mit $+0,5 \mathrm{D}=5 / 5$.

Wenn die multiplen Hauttumoren nicht gewesen wären, hätte ich wegen der entzündlichen Begleiterscheinungen die Neubildung am rechten Augenwinkel für eine Dacryoadenitis gehalten und incidirt. - So aber beschränkte ich mich bei dem höchst frappanten sonstigen Krankheitsbild vorläufge auf Verordnung indifferenter Umschläge auf das Auge und allgemein diätetischer Rathschläge im Uebrigen.

Am 21. IX. erweist sich ein grosser Theil der Tumoren, trotz der kurzen inzwischen verflossenen Zeit, colossal vergrössert. Schon von Weitem sichtbar machen einzelne, unter dem tastenden Finger, den Eindruck der Grösse von Wallnüssen. Besonders imposant erseheint eine Geschwulst, die pilzartig der rechten Wange aufsitzt. - Auch scheinen an Brust und Rücken ete. jetzt ebenso viel Tumoren wie früher im Gesicht zu sitzen. Sie sind aber immer noch vollständig beweglich und von normal aussehender Haut bedeckt. Ebenso sind die Lymphdrüsen zweifellos vergrössert, treten aber im Krankheitsbilde neben den Hautgeschwülsten vollständig zurück.

Das Allgemeinbefinden ist wie früher.

Der Tumor am rechten äusseren Augenwinkel ist ebenfalls erheblich gewachsen. Er wölbt jetzt nicht nur die Lidhaut, sondern auch die Conjunetiva der oberen und unteren Uebergangsfalte aussen, sowie das Ligamentum palpebrae externum erheblich vor, so dass bei ektropionirtem Oberlid die von der gerötheten Bindehaut bedeckte Kuppe der Geschwulst genau betrachtet und betastet werden kann, allerdings ohne dass dadurch Neues zu Tage gefördert wird. 
Einen ganz anderen Eindruck dagegen wie am 13. IX. machen die Uebergangsfalten. Ihre Oberfläche ist vorgewölbt, sulzig, glanzlos und von leicht höckriger Beschaffenheit. Ihr Aussehen ist durchaus anders wie bei Trachom oder Follicularkatarth, oder Amyloid, oder sonst irgend einer mir bis dahin zu Gesicht gekommenen, oder sonst beschriebenen Conjunctivalveränderung.

Sucht man yon aussen durch die Lidhaut hindurch die Gegend der Uebergangsfalte zu betasten, so fühlt man in der Tiefe eine wurstähnliche, vom äusseren bis zum inneren Lidwinkel reichende Geschwulst von ziemlich fester Consistenz und der ungefähren Dicke der stärksten Thränensonde. Auch in dieser Beziehnng muss ich sagen, dass ich noch nicht entfernt etwas Aehnliches bei irgend einer Conjunetivalerkrankung bemerkt habe und auch keines der mir bekannten Krankheitsbilder zum Vergleich heranzuziehen wüsste.

Die gleichen, wenn auch nicht so stark ausgeprägten Veränderungen sind anch am Oberlid nachweisbar und - am linken. Auge. Auch hier ist heute eine allerdings noch sehr kleine Geschwulst am äusseren Augenwinkel bemerkbar, ebenso wie die Uebergangsfalten eine Erkrankung zeigen, die vollständig der am 13. IX. rechts constatirten gleicht.

Von dem Gewebe der unteren Vebergangsfalte rechts wird ein kleines Stiick excidirt, ebenso unter localer Anästhesie durch Herrn Dr. Staffel ein haselnussgrosser Tumor aus der Brusthaut.

Leider wird versäumt ein Blutpräparat anzulegen, was später wegen der strikten Weigerung der Patientin, noch irgend etwas mit sich machen zu lassen, nicht nachgeholt werden konnte.

Herr Dr. Staffel, der die Patientin an diesem Tage zum ersten Mal sah, glaubte soforl auf Grund der Anamnese und des soeben beschriebenen Befundes, dass die Diagnose Mycosis fungoides die wahrscheinlichste sei.

Von den in Alkohol gehärteten Gewebsstücken wurden nach Einbettung in Celloidin sofort Schnitte angefertigt, die nach den verschiedensten Methoden gefärbt und den Herren Prof. Aschoff und Unna zugesandt wurden. Die beiden Herren brachten den Präparaten das gxösste Interesse entgegen und hatten die Güte, die an sie gerichteten Fragen eingehend zu beantworten, wofür ich ihnen auch an dieser Stelle meinen verbindlichsten Dank sage.

Schwanken könne man mit Rücksicht auf den bisherigen klinischen Verlauf, der noch nicht zur Bildung von Eczemen und Ulcerationen geführt habe, hauptsächlich zwischen der Diagnose multiple Sarkome und Mycosis fungoides.

Auch gäbe die histologische Untersuchung vorläufig keine absolut zweifellosen Anhaltspunkte zur sicheren Entscheidung für die eine oder die andere Möglichkeit. Für Sarkome spreche das Freibleiben des Papillarkörpers von Geschwulstzellen und der Mangel an Epithelveränderungen der Haut. Alles andere jedoch weise auf Mycosis fungoides hin, so vor Allem der an Infiltration erinnernde Aufbau der Geschwulst, der Reichthum an Plasmazellen und die für ein Randzellensarkom viel zu grosse Polymorphie der Zellen; ferner der Umstand, dass dieselben sehr schlecht contourirt seien und an den Rändern äussersst unregelmässige Kanten und Leisten von 
Granoplasma trügen; schliesslich der Kernreichthum und die vielen multiplen Kerne. - Wenn sich nun auch in den Lymphspaiten noch nicht die für Mycosis fungoides so charakteristische Ansammlung von Granoplasmakörnchen constatiren liesse, so erkläre sich dies aus der Krankengeschichte. Der Tumor befinde sich noch im Stadium Incrementi, während der reichliche Zerfall des Protoplasmas erst später aufträte. - Die Form der Mycosis fungoides, um die es sich wahrscheinlich handle, sei nicht die flechtenartig beginnende, sondern die mit sarkomähnlichem Habitus.

Als Therapie werden jetzt neben roborirender Diät starke Dosen asiatischer Pillen verordnet, sowie Einträufelungen schwacher Cocainlösungen in die Conjunctivalsäcke, da die subjectiven Beschwerden der Augen nicht besser geworden sind.

28. IX. Eine erhebliche Vermehrung der Hautgeschwïlste hat nicht mehr stattgefunden. Auch sind die am 21. IX. als sehr gross imponirenden Tumoren nicht noch weiter gewachsen, wohl aber hat eine Anzahl der trüher klein erseheinenden jetzt ebenfalls den Umfang von Wallnüssen erreicht. Besonders triffit dies zu für den Rücken.

Die Haut ist überall normal, das Allgemeinbefinden wie früher. -

Der Tumor in der Gegend der rechten Thränendrüse ist seinem Umfang nach ebenfalls wie früher, ebenso die Infiltration der Vebergangsfalten. Es hat sich ein leichtes Entropium des Unterlides ausgebildet, so dass die Cilien auf die Cornea reiben und oberflächliche Substanzverluste verursachen.

Links hat die Affection der Thränendrïse and Uebergangsfalten zugenommen, jedoch ist hier bei Weitem noch nicht der Status des rechten Auges erreicht.

10. X. Das Allgemeinbefinden ist schlechter geworden. Die Patientin hat meist gelegen. Appetit hat nachgelassen. Puls 80, kleiner als fither. Temperatur Abends gewöhnlich 37,3. - Urin frei von Eiweiss und Zucker.

Haut und Tumoren des Gesichtes, der Brust und Extremitäten sind im Allgemeinen wie früher. An einzelnen Stellen jedoeh bekommt man den Eindruck, als ob sie kleiner geworden wären. Das Gleiche gilt von den Geschwïlsten auf dem Rücken. Hier aber fällt auf, dass sie gegen die bedeckende Haut nicht mehr so verschieblich sind wie früher. Auch hat die Haut an den Stellen der Vorwölbung ein röthlich-blaues Aussehen angenommen, so dass ein ganz ausserordentlich imposantes Krankheitsbild zu Stande gekommen ist.

Die Lymphdrüsen sind im Allgemeinen jetzt viel stärker vergrössert als früher. Sie erreichen zum Theil jetzt den Umfang von Pflaumen.

Am rechten Ange hat die Infiltration der unteren Uebergangsfalte eher abgenommen, wohl aber hat sie sich unter der Conjunctiva sclerae ausgebreitet, so dass auch hier dasselbe miraculöse Aussehen wie an der Uebergangsfalte zu constatiren ist. Es ist nur ein ca. $2 \mathrm{~mm}$ breiter Saum bis zum Hornhautrande frei geblieben.

20. X. Das Allgemeinbefinden ist nicht gebessert. Abends wurden meist Steigerungen der Temperatur bis za $38^{\circ}$ registrirt.

Aber - die subcutanen Geschwülste sowohl wie die Drüsentumoren sind zweifellos verkleinert!

Am wenigsten auf dem Rücken, wo sich jetzt an den blaurothen 
Stellen vielfach ein oberflächliches, leicht nässendes Eezem gebildet hat, das in seinem Aussehen zum Theil an Pityriasis versicolor erinnert.

Am rechten Auge ist ebenfalls eine geringe Verkleinerung des Thränendiüsentumors und des Bindehautinfiltrates eingetreten. - Am linken Auge ist schon jetzt kaum noch etwas Pathologisches zu sehen.

1. XI. Das Allgemeinbefinden wird immer schlechter. Puls 92, Temperaturen bis $38,2^{\circ}$. Leichte Ohnmachtsanfälle sind aufgetreten. Patientin kann das Bett nicht mehr verlassen. - Arsen wird ausgesetzt.

Im Gesicht sind die Tumoren zum grossen Theil verschwunden, eine Anzahl von Bohnengrösse ist jedoch noch zu fühlen. Das Gleiche gilt von Hals, Extremitäten und Rüeken, wo das jüngst bemerkte blau-röthliche Aussehen mancher Hantpartien sowie das Eczem grösstentheils nicht mehr vorhanden ist. Auch scheint die Haut über dem Tumor wieder voliständig beweglich zu sein. Auf der Brust ist ebenfalls alles verkleinert, jedoch sind hier jetzt auch an einzelnen Stellen blau-rothe Flecken in der Haut zu sehen, die über haselnussgrossen Tumoren liegen.

In gleicher Weise sind die Drüsentumoren bis auf einzelne wenige in der Inguinal- und Axillargegend verschwunden.

Die Geschwulst im rechten Augenwinkel ist nicht mehr zu sehen. Ebenso ist von der Conjunetivalaffection ausser dem eigenartigen Aussehen nur noch eine minimale eben noch fühlbare Verdickung der Uebergangsfalte nachweisbar.

28. XI. Das Aussehen der Patientin erinnerte inzwischen immer mehr an Krebskachexie. Die Temperaturen blieben in derselben Höhe, der Puls wurde langsam kleiner und stieg bis 125. Dazu kamen leichte Oedeme. Exitus letalis.

Von den Tumoren waren schliesslich mit Sicherheit nur noch zwei oder drei zu fühlen unter der Brusthaut und am rechten Oberschenkel, ebenso nur einzelne Drüsengeschwülste in der rechten Leistenbeuge. Einzelne leicht blau-rothe Stellen waren jedach noch übrig geblieben.

Bei der Section, die leider erst zwei Tage post mortem vorgenommen werden konnte, fanden sich in der Brusthöhle ziemlich ausgedehnte pleuritische Verwachsungen, sowie Verdickungen an den Mitral-, Aorten- und Pulmonalklappen. Die Aorta zeigte sehr geringe arteriosklerotische Veränderungen.

An den Organen des Abdomen vermochte ich nur wenige Abnormitäten zu constatiren. Erstens fand sich eine ca. 50 Pfennigstück grosse, gelb-weissliche Verfärbung in der linken Nierenrinde, zweitens im unteren Theil des Oesophagus eine oberflächliche Geschwürsfläche mit gezackten Rändern und etwas zerklüftetem Grunde, schliesslich schienen einzelne Mesenteriallymphdrüsen etwas vergrössert.

Die Milz fühlte sich ziemlich derb an, war von blau-rother glänzender Oberfläche und mit drei bis vier Einkerbungen versehen. Sie mass im grössten Durehmesser $11 \mathrm{~cm}$ und wog frisch aus der Leiche genommen etwas weniger als $130 \mathrm{~g}$. - Auf der Schnittfläche erschien die Pulpa blutreich, tief dunkelroth und die Malpighi'schen Körperchen weiss-gelblich, höehstens $1 \mathrm{~mm}$ im Durchmesser gross. 
Die Leber wog $3^{1 / 2}$ Pfund und war von glatter, leicht glänzender Serosa überzogen. Die Schnittläche war im Allgemeinen von bräunlicher Farbe, die centralen Theile der Acini waren röthlich, die peripheren mehr gelb. An der Glisson'schen Kapsel oder an den Gefässwandungen waren keinerlei Verdickungen oder Infiltrationen $\mathrm{zu}$ constatiren.

Die unter der Haut der Brust und des rechten Oberschenkels fühlbaren Tumoren wurden exeidirt mit der lose darüberliegenden, blau-röthlich verfärbten Haut, ebenso die drei vergrösserten Inguinaldrüsen. -- Die letzteren sehen auf der Schnittfäche gleichmässig blass-grauroth aus, die Hanttumoren im Centrum mehr weiss-gelblich.

Das Sternum und die Rippen liessen sich mit dem Knochenmesser nur unter Anwendung einer gewissen Gewalt schneiden. Das sichtbare Mark war von lebhaft röthlicher Farbe.

Schliesslich wurde vom Cranium aus die ganz in Fettgewebe verhüllte Thränendrüse des rechten Auges herausbefördert, sowie ein grösseres Stïck der unteren Uebergangsfalte. Die erstere erschien jetzt doch noch erheblich vergrössert, vielleicht um die Hälfte.

Bei der Beurtheilung der nach den versehiedensten Methoden behandelten Präparate hatten die Herren Proff. Aschoff und Unna wiederum die grosse Gute, mich mit ihrem Rath zu unterstützen.

Die gelbliche Verfärbung in der Nierenrinde erwies sich als anämischer Fleck, wie er ofter in Folge postmortaler Blutstaunngen und Druckes auf die Organe bei der Section der Leber und Nieren angetroffen wird. Als pathologisehe Veränderung fanden sich nur einzelne kleinzellige Herde, wohl als Ausdruck einer leichten interstitiellen Nephritis.

Das Geschwür in der Speiseröhre war ebenfalls durch postmortale Maceration des Epithels entstanden. In einzelnen Schnitten fanden sich Drüsenkörper mit Anhäufung lymphatischen Gewebes um die ampullenförmig aufgetriebenen Ausführungsgänge, sonst nichts Pathologisches.

Ueber die Milz schrieb Herr Prof. Aschoff Folgendes: ${ }^{\text {Sie }}$ zeigt starke Hyperämie, geringe Pigmentablagerungen, im Uebrigen normales Gefüge und nichts was auf Leukämie hinweist. Auffallend sind aber verschiedene kleine knötchenförmige, in der Pulpa gelegene, wie Tuberkel grosse Herde, die aus reticulärem Bindegewebe mit dichter Einlagerung von Rundzellen bestehen und in denen sich frische Fibringerinnungen mit rothen Blutkörperchen im Fibrinnetz befinden. Aeltere Processe sind es nicht, da jegliches Pigment fehlt. Für lenkämische Veränderangen sind sie zu geringfiugig. Leider ist die Kernstructur der Zellen nicht genügend zu erkennen. Tuberkel sind es auch nicht, auch keine Abscesse, vielleicht kleine infectiöse Granulome anderer Natur. Ich glaube nicht, dass sie mit der Hautkrankheit in Beziehung stehen. Amyloid ist ebenfalls nicht nachweisbar."

Ueber die Lymphknoten schrieb er: "Diejenigen aus dem Mesenterium zeigen das Bild normaler Lymphknoten, ebenso die kleinen neben dem grossen Inguinaltumor sitzenden.

An dem letzteren findet sich an einer Stelle eine die Kapsel weithin tiberschreitende, bis in das Fettgewebe vordringende Wucherung. Die Zellen derselben sind ebenso vielgestaltig wie die in den Hautschnitten, enthalten 
aber keine typischen Plasmazellen. Die Kernstructur ist nicht hinreichend deutlieh. In der Umgebung der Wucherung liegt Pigment. - Der Lymphknoten selbst enthält sehr viel Plasmazellen. Es ist auch deutliche Blutresorption zu sehen, ferner Pigment in den Endothelzellen der Lymphsinus, auch scheinen blutkörperchenhaltige Zellen da zu sein. Endlich finden sich vereinzelte Mastzellen und Russel'sche Körperchen, die aber nichts Charakteristisches besitzen.

Die Lymphknoten zeigen eine gewisse Hyperplasie, einen ehronischen Reizzustand, aber kein für maligne Lymphombildung beweisendes Bild: Sichere Anhaltspunkte für die Diagnose Leukämie oder Pseudoleukämie sind demuach nicht zu finden. - Etwas Bestimmtes kann aber aus dem histologischen Befunde allein nicht geschlossen werden."

Die in Alkohol gehärteten und in Celloidin eingebetteten Gewebsstücke mit den Hauttumoren boten, makroskopisch betrachtet, auf der Schnittfläche genau das Bild, das Neisser ${ }^{\mathrm{y}}$ ) als für Myeosis fungoides charakteristisch beschreibt. Unterhalb der in diesem Falle allerdings unversehrt erscheinenden Cutis lagen die weiss-gelblichen Geschwulstmassen ohne scharfe Begrenzung gegen die Umgebung, sondern nach allen Richtungen hin mehr oder weniger lange, im Fett sich allmählich verlierende Fortsätze aussendend.

Bei der mikroskopischen Untersuchung fand sich, dass die Geschwulst aus dicht an einander liegenden Zellen bestand, deren ausserordentliche Polymorphie sowohl in Bezug auf die Grösse, als die Gestaltung des Kernes und des Protoplasmaleibes sofort auffiel. Nur die Spindelform fand sich nicht vertreten. Die Polymorphie war jetzt entschieden noch frappanter als auf den Schnitten durch das intra vitam excidirte Stick. Kerntheilungsfiguren fanden sich dagegen viel weniger als auf den letzteren, wo sie stellenweise massenhaft zu sehen waren. Das Cytoplasma war, wie man an den Methylenblaupräparaten besonders deutlich sehen konnte, vielfach bis auf kleine Reste verschwunden, so dass manche Kerne oder auch Kernkörperchen als kugel- oder halbmondförmige, verklumpte Gebilde umher lagen.

Bakterien fanden sich nirgends.

Die Unna'schen Zellbrocken in den Lymphspaiten liessen sich nicht sicher nachweisen, wenugleich mír schien, als ob einzelne blau gefärbte Körnchen als solche gedeutet werden könnten. Herr Prof. Unna meinte, dass die Darstellung derselben durch die chemische Einwirkung von Tannin, das aus dem Kork atisgelaugt und in das Gewebe gedrungen sei, verhindert würde.

Ebenso sei dadureh der Nachweis von Plasmazellen erschwert, trotzdem sicher sei, dass dieselben zahlreich vorhanden wären, wie man aus der Lage der Kernkörperchen im Kern vieler Zellen annehmen müsse. Mastzellen, deren Protoplasmakörner sich mit polychromem Methylenblau roth gefärbt hatten, waren massenhaft vorhanden, sowohl in den ante als post mortem gewonnenen Gewebsstücken.

Ein auffallender Untersehied zwisehen denselben war aber der, dass bei den letzteren colossal viel eisenhaltiges Pigment sich angehäuft hatte meist innerhalb der Geschwulstzellen, deren Kern oft gänzlich dadurch ver-

1) Krankheiten der Haut. Handb. d. prakt. Med.v. Ebstein u. Sch walbe. 1900. 
deckt war. Das meiste Pigment lag in der Nähe der Gefässe, aber auch an anderen Stellen, an denen man nicht einmal Reste derselben erkennen konnte. Eigentliche nekrobiotische Erscheinungen zeigten sich nirgends.

Ein neugebildetes Stroma innerhalb der Geschwulst existirte nicht, noch viel weniger lymphatisches Gewebe als Gerüst. Die vielgestaltigen Zellen waren vielmehr eingelagert zwisehen die Bindegewebsfasern und Balken, die zwar aus einander gedrängt und comprimirt, aber zum grössten Theil noch erhalten waren (Prof. Aschoff), wie man besonders deutlieh an den post mortem gewonnenen Schnitten sehen konnte. Die nach van Gieson und mit Oreeïn behandelten Präparate waren in dieser Beziehung besonders instructiv. Die elastisehen Fasern zogen vielfach intact durch die Geschwulst hindurch.

Wenn man die peripheren, aus den mit blossem Auge sichtbaren Fortsätzen bestehenden Theile der Geschwulst betrachtete, hatte man überbaupt den Eindruck, als ob das ganze krankhafte Gewebe rerschwunden sein würde, wenn nicht die Patientin gestorben wäre. Die Fortsätze bestanden zuerst aus Zellen, die zwei- bis dreireihig in den Lymphspalten zwischen den Fettzellen lagen, ein netzförmiges Maschengewebe mit einander bildend. Weiterhin lagen sie nur noch einreihig, schliesslich vollständig vereinzelt und zerstreut, meist mit Pigment vollgepfropft, so dass man annehmen musste, sie seien bei dem Resorptionsprocess liegen geblieben. In diesen Gegenden fanden sich hier und da auch Fibroblasten mit langgestreckten Fortsätzen, vielfach unentwirrbare Knäuel bildend.

Innerhalb des bei oberflächlicher Betrachtung noch compact erscheinenden Inneren des Tumors waren übrigens ebenfalls Lücken vorbanden von meist rundlicher Gestalt, die offenbar durch Schwund von Gesehwulstmassen entstanden waren, da die sie begrenzenden Zellen ïberall wegen ihres zernagten, unregelmässig contourirten Randes die Spuren des Zerbröckelns an sich trugen.

In der Richtung nach der Musculatur waren die soeben beschriebenen Ausläufer vielfach nicht zu finden, hier bildete meist ein dicker Bindegewebsstrang einen, wie es schien, soliden Abschluss.

Nicht so scharf war aber die Grenze nach der eigentlichen Cutis zu. Dieselbe war jetzt doch nicht mehr so intact wie bei dem intra vitam excidirten Gewebsstück. Hier und da sah man um die Schläuche der Schweissund Talgdrüsen Zellinfiltrationen derselben Art, wie im eigentlichen Tumor. Eine Zerstörung der drüsigen Elemente fand sich jedoch nicht.

Die Epidermis war normal.

Dasjenige, was ïber die histologische Beschaffenheit der Hauttumoren gesagt ist, hat im Allgemeinen auch für die Infiltrationen in das subconjunetivale Gewebe Geltung. Die Polymorphie der Zellen war auch hier frappant, vielleicht noch mehr als bei den erst beschriebenen, nur lagen sie nieht so dicht beisammen. Die Abbröckelungserscheinungen waren ebenfalls sichtbar, Mastzellen fanden sich geradezu massenhaft in jeglicher Grösse und Form, ebenso an den frischen Methylenblaupräparaten Plasmazellen.

Von einer Zerstörung des Grundgewebes war auch bei der Conjunctiva keine Rede. Die Zellen schoben sich vielfach rosenkranzähnlich zwischen die Bindegewebsfasern hinein, dieselben aus einander drängend. Zablreiche Lymphspalten waren vorhanden und viele normal erseheinende Gefässe, 
von denen nur wenige mit geschwulstzellenhaltigen Thromben verstopft erschienen.

Sehr wenig Kernthellungsfiguren fanden sich sowohl in dem frisch excidirten als auch in dem später gewonnenen Stück.

An dem letzteren konnte man sehen, dass die krankhaften Veränderungen nur in dem lockeren Gewebe unmittelbar unter dem Conjunctivalepithel sassen, während mit der äusseren Lidhaut kein Zusammenhang bestand. Die oberen Schichten des Epithels selbst waren meist verschwunden, so dass von demselben vielfach nur noch eine Zellreihe sichtbar war, eine Uleeration war jedoch nicht vorhanden.

An den Grenzen der Infiltration fanden sich massenhaft Fibroblasten, viel mehr als unter der Cutis, so dass der entzündliche Charakter des Ganzen an der Conjunetiva noch mehr als dort hervortrat. Man hatte an manchen Stellen geradezu den Eindruck, als ob eine Abkapselung vor sich gehen sollte. Eisenhaltiges Pigment war verhältnissmässig nur wenig nachzuweisen. Auch sah die Grenzschicht des Tumors unter der Bindehaut insofern etwas anders aus, als die Resorption offenbar mehr gleichmässig vor sich ging, so dass die oben beschriebenen netzförmigen Zellconglomerate nicht in dieser Ausdehnung zu Stande gekommen waren.

In dem die vergrösserte Thränendrüse enthaltenden Fettgewebe fand. sick wiederum eine Zellanhäufung von der gleichen Qualität. Das Gewebe der Drüse war eingebettet in die Geschwulstmassen, die an einzelnen Stellen, an welchen sie durch die Kapsel begrenzt wurden, mehr den Eindruck eines circumscripten Tumors machten, an anderen dagegen, wo sie bei der Fortwucherung sich nicht an dieselbe gekehrt hatten, mehr einem Infiltrat ähnlich sahen.

Vor Allem war zu bemerken, dass die Acini manchmal weit aus einander gedrängt waren, dass aber nirgends das Epithel oder Stützgewebe mit Sicherheit als zerstört bezeichnet werden konnte.

Ganz besonders schön und in jeder Gestalt vorhanden waren in der Umgebung der die Acini ernährenden Blatgefässe Plasmazellen, manchmal so dicht, dass fast das ganze Gesichtsfeld der Immersionslinse aus denselben bestand. Selbstverständlich fanden sich auch viele Mastzellen.

Auffallend an einigen Schnitten durch die Thränendrüse waren starke Zellinfiltrationen in der Umgebung einzelner grösserer Gefässe, die bei den Präparaten von den anderen Organen nicht in dieser Weise hervortraten. Auch war die Polymorphie an vielen Stellen nicht so sehr ausgebildet. Bezüglich der Fibroblasten und des Pigments kann das bei der Conjunctiva Gesagte wiederholt werden.

Epikrise.

Wir wollen den vorliegenden Fall, nachdem er in seinem ganzen Verlaufe vor uns aufgerollt ist, zunächst vom rein klinischen Standpunkt aus zu differenziren suchen. Folgende Möglichkeiten würden dann wohl in Betracht zu ziehen sein: Multiple Hautsarkome, Sarkoide, Leucaemia und Pseudoleucaemia cutis, Lymphodermia perni- 
ciosa cutis und Mycosis fungoides, alles Krankheitsbegriffe, die bezüglich ihres Verhältnisses zu einander, ihrer Symptome, Aetiologie und Histologie zu einem der dunkelsten Gebiete der Pathologie überhaupt gehören dürften.

Dermatologische Autoritäten wie Unna, Kaposi, Neisser, deren Arbeiten als Beispiele aus der umfangreichen, hier nicht vollständig zu erörternden Literatur herausgegriffen sein mögen, geben dies ohne Weiteres zu.

Neisser (loc. cit. $)^{1}$ ) stellt die Mycosis fungoides, Lencaemia, Pseudoleucaemia und Lymphodermia perniciosa cutis in einem Capitel zusammen, „da vom klinischen Gesichtspunkt aus zweifellos die Berechtigung zu dieser Vereinigung bestehe und auch dem pathologischanatomischen Befunde nach; denn die hierher gehörigen Affectionen seien offenbar nahe verwandt, da es sich bei fast allen um rundzellige Neubildungen handle". „Im Uebrigen wiese man ihnen gewohnheitsmässig eine Stellung an zwischen den an und für sich weit verschiedenen wirklichen Tumoren und entzündlichen Neubildungen, da sie Erscheinungen böten, die für jede der beiden als charakteristisch angesehen würden."

Neisser glaubt, dass die vier Krankheitsbilder wegen verschiedener Eigenthümlichkeiten von einander zu trennen wären, und dass auch meist der einzelne Fall unter eines der vier Schemata rubricirt werden könne.

Der vorliegende würde dann zweifellos am besten unter die $\mathrm{My}$ cosis fungoides passen, allerdings nicht wegen seines rasend schnellen Verlaufes unter die gewöhnliche Form, sondern unter die Vidal'sche Gruppe ${ }^{8}$ ): die Mycosis fungoides d'emblée. Bei dieser fehlt das Jahre lang danernde eczematöse Prodromalstadium, und „aus gesunder Haut schiessen plötzlich pilzartig Tumoren empor, erodiren, ulceriren oder verschwinden wieder und bieten so zu sagen nur das Endspiel, das letzte Stadium isolirt dar".

Wegen der Lymphdrüsenschwellung, die bekanntlich bei Mycosis fungoides nur selten vorkommt, muss man darauf zunächst an eine etwa primär vorhandene Pseudolencaemia und Leucaemia lymphatica denken (Biesiadecki, Hochsinger und Schiff, Besnier, Vidal, Joseph, Arning, E. Wagner, Kreibisch etc.). Die Drïsenschwellung trat aber im Verlaufe der Affection, wie die Kranken-

1) In Gemeinschaft mit Pincus.

2) Vidal u. Brocq, Étude sur le Mycosis fungoides. La France médicale. 1885. p. $79-85$. 
geschichte zeigt, so vollkommen zurück gegen die Hauttumoren, ja sie machte so vollständig den Eindruck des Secundären oder Metastatischen, dass schon aus diesem Grunde jene Annahme unwahrscheinlich wird. Zu erinnern wäre hierbei auch an Winiwarter's Abhandlung in der deutschen Chirurgie ${ }^{1}$ ), der über die Hauttumoren der Psendoleucaemia und Leucaemia schreibt, dass ,sie nur als Ausdruck einer sehr verbreiteten intensiven Erkrankung vorzukommen scheine, ohne eine praktische Bedeutung zu erlangen".

Wenn man Neisser's ausserordentlich klarer Darstellung folgt, kann man aber aus verschiedenen rein klinischen Symptomen unseren Fall ganz gut differentialdiagnostisch gegen die an und für sich sehr ähnlichen Krankheitsbilder der Leucaemia cutis etc. abgrenzen, auch ohne Heranziehung der leider versäumten Blutuntersuchung, die nach ihm hauptsächlich für die beginnenden Fälle Bedeutung hat: Vor Allem sollen die Tumoren der Pseudoleucaemia und Leucaemia cutis immer mit der Hautdecke fest verwachsen sein, so dass dieselbe über dem Tumor nicht abhebbar ist, während sie sammt der darunter liegenden Geschwulst gegen die Unterlage des Ganzen verschoben werden kann. Die Haut selbst soll auffallend durchsichtig und von lang hingestreckten, dicken Blutgefässen durchzogen sein, was alles für unseren Fall nicht zutrifft.

Das Gleiche gilt für die sehr oft bei den pseudoleukämischen Hauttumoren auftretenden Juckausschläge, die in unregelmässig verbreiteter Urticaria papulosa oder in Blasenbildungen bestehen.

Wit der Lymphodermia perniciosa hat unser Fall am Wenigsten zu thun. Es fehlt der universell eczematöse Zustand, die teigige Schwellung der Haut, die Erythrodermia und schliesslich nach Ausbruch der Tumoreu das Leontiasis ähnliche Aussehen der Kranken. -

Nach Kaposi ${ }^{2}$ ) ist es ,bisher nicht gelungen, weder auf Grund des histologischen Befundes, noch der klinischen Charaktere, noch der Berücksichtigung beider, ein abschliessendes Urtheil über die pathologische Bedeutung (sc. der Mycosis fungoides), ja selbst auch nur eine strikte klinische Abgrenzung gegenüber den übrigen zur Gruppe der sarkoiden Geschwïlste gehörenden Krankheitstypen zu gewinnen".

Ob Hautgeschwülste von dem Charakter der Mycosis fungoides, welche unter leichten Fiebererscheinungen schnell entstehen und vergehen können, „mit Hinterlassung vollkommen unveränderter, nur mehr

1) Winiwarter, Die chirurgisehen Krankheiten der Haut und des Zellgewebes. Liefg. 23.

2) Pathologie und Therapie der Hautkrankheiten. 1899. 
oder weniger pigmentirter Cutis", Beziehungen zu Leukämie oder Pseudoleukämie haben können, hält er mit Paltauf überhaupt noch nicht für nothwendig, sondern nur für möglich. Er hält deshalb auch die Meinung Paltauf's für sehr beachtenswerth, der diejenigen Fälle als eine Abart der Mycosis fungoides auffasst, welche durch Complication mit Lymphdrüsenschwellung, Milztumor, Leberschwellung „,bei einer neben der Anämie einhergehenden leukocythämischen Beschaffenheit des Blutes, dem Vorkommen solcher Knoten (Lymphome, Lymphosarkome) am harten Gaumen, an den Tonsillen, das Bild von Pseudoleukämie und Lymphadenie darbieten".

Nach Kaposi würde man unseren Fall, mit Bezug auf die geschwollenen Lymphdrüsen, also ziemlich unangefochten zur Mycosis fungoides rechnen können. -

Aber jetzt fragt sich, wie sein Verhältniss zur Sarcomatosis cutis ist und ob er von der letzteren durch seine klinischen Erscheinungen differenzirt werden kann. Die grösste Schwierigkeit bei Lösung dieses Problems liegt wiederum in der grossen Unklarheit, welche über die Hautsarkomatose herrscht. Wie soeben angedeutet, rechnet sie Kaposi neben der Mycosis fungoides und Lymphodermia perniciosa zu den sarkoiden Geschwülsten. Besonders aus klinischen Gründen sei aber eine Unterscheidung gegen die beiden soeben genannten Krankheitsformen nicht nur geboten, sondern auch möglich. „Während bei der multiplen Sarkombildung regelmässig die tieferen Schichten des Corium und die Subcutis bei langem Freibleiben der Papillarschicht von dem Zellinfiltrat betroffen würden, sei der hauptsächliche manchmal ausschliessliche Sitz der Veränderungen bei der Mycosis fungoides in der obersten Cutis- und Papillarschicht." Er hält dies Unterscheidungs" merkmal offenbar aber nicht für durchführbar, denn sonst könnte er nicht an einer anderen Stelle sagen: "Zweifellos sind manche in der Literatur als Mycosis fungoides bezeichnete Fälle auch im Sinne der Sarkomatosisformen deutungsfähig und umgekehrt."

Neisser behandelt die Hautsarkome unter den "malignen Neoplasmen" in einem Capitel für sich und theilt sie, wie mir scheint, in ausserordentlich zweckmässiger Weise in die eigentlichen Hautsarkome, welche die den malignen Geschwülsten zukommenden Attribute des unbeschränkten Wachsthums und der Metastasenbildung besitzen, und in diejenigen, ,welche nicht nach allen Richtungen hin dem bekannten Sarkomtypus entsprechen, aber vorläufig auch sonst noch nirgends unterzubringen sind und daher den provisorischen, von verschiedenen Autoren allerdings noch sehr verschiedenartig aufge- 
fassten Namen der, sarkoiden Geschwülste bekommen haben und vorläufig behalten können".

Die sarkoiden Geschwülste zerfallen nach $\mathrm{N}$ eisser in zwei Theile: 1. in das typische, idiopathische, multiple, haemorrhagische Sarkom (Acrosarkom Kaposi's), das für unseren Fall ebenso wenig wie das eigentliche Hautsarkom in Betracht kommen kann, 2. in die atypischen Fälle. "Bei letzteren handelt es sich meist um Rundzellentumoren, deren Abgrenzung von der Pseudoleukämie und von den eigentlichen Rundzellensarkomen zur Zeit noch nicht möglich ist, oder um Tumoren (mit und ohne Betheiligung der inneren Organe), bei denen die fehlende Schrankenlosigkeit des Wachsthums, der mehrmals benigne Verlauf (Heilung durch Arsen, durch Erysipel etc.) und das histologische Bild (Einlagerung der Geschwulstzellen in die Lymphspalten der Cutis mit Erhaltung der Structur der letzteren) zu $Z$ weifeln an der echten Sarkomnatur Anlass giebt."

An diese Fälle hat Neisser jedenfalls auch gedacht, wenn er bei Besprechung der Mycosis fungoides d'emblée sagt: „Diese letztere Form führt zu dem klinischen Bilde einer Art der sog. Hautsarkome über, einer Neubildungsform, welche aus Rund- oder Spindelzellen besteht und mit der Mycosis fungoides sogar die Eigenschaft des spontanen oder therapeutisch erzeugten Schwundes gemeinsam hat."

Sehr oft in der betreffenden Literatur angeführte Beispiele für die atypischen Sarkoide bieten die Fälle Spiegler's 1). Bei denselben handelte es sich um Hauttumoren ron dem Charakter der Sarkome, die aber verschwanden und unter leichten Fiebererscheinungen zum Tode führten. Die blutbereitenden Organe waren normal. Spiegler hält die Geschwülste für Uebergangsformen von entzündlicher Wucherung zu echten Sarkomen. - Ein handgreiflicher Unterschied der betreffenden Krankengeschichten gegen das Bild der Mycosis fungoides d'emblée ist eigentlich nicht zu finden. Auch ist ihre Aehnlichkeit mit der unsrigen zum Theil gross. Einmal begann die Affection sogar mit einer später spurlos wieder verschwindenden Geschwulst im linken äusseren Augenwinkel.

Aus dem Gesagten geht jedenfalls hervor, dass unser Fall durch seine klinischen Merkmale zwar ganz gut gegen die Pseudoleucaemia cutis etc. abgegrenzt werden kann, nicht aber gegen die Hautsarkome, so dass in dieser Beziehung der anatomische Befund den Ausschlag geben muss.

1) Arch. f. Dermat. u. Syphilis. 1894. S. 163. 
Aber leider herrscht unter den Dermatologen wiederum keine einheitliche Anschauung über die anatomischen Eigenthümlichkeiten der Hautsarkome und der Mycosis fungoides.

Das markante histologische Charakteristicum der Sarkoide ist nach Kaposi, Spiegler, Neisser und Anderen die vorhin schon angedeutete Thatsache, dass das von dem Infiltrat eingenommene Gewebe nicht wie beim bösartigen Tumor sensu strictori zerstört, sondern erhalten wird, so dass das Stroma der Geschwülste aus dem unveränderten Maschenwerk der Cutis besteht, welches in ziemlicher Unversehrtheit nach Verschwinden der Geschwulstmassen bei der Section sich wiederfindet.

Unna ${ }^{1}$ ) classificirt in seinem grossen Werke die Hautsarkome bekanntlich nach anderen Principien. Einen histologischen Unterschied nach der eben angedeuteten Richtung hin scheint er zwar zwischen verschiedenen Formen von Rundzellensarkomen zu machen, er zieht aber daraus nicht jene tief greifenden Consequenzen für die klinischen Eigenschaften derselben. $\operatorname{Er}$ giebt nämlich an, dass bei gewissen seltener vorkommenden Sarkomen durch Auspinselung ein collagenes Gerüst von gröberen und feineren Balken, das keine lymphoide Structur besitze, sichtbar gemacht werden könne, in welchem das elastische Gewebe erhalten bliebe. Uebrigens giebt er zu, dass „die klinischen und anatomischen Kenntnisse der Hautsarkome noch nicht so weit gediehen sind, um bereits jedem der angeführten Typen ein in sich abgeschlossenes histologisches Bild zu Grunde zu legen".

Weiterhin legt Kaposi, Spiegler und, wie es scheint, auch Neisser ein grosses Gewicht auf die hämatogene Pigmentirung der Sarkoide, die in gewissem Grade charakteristisch für dieselben sei, während Unna in allen Arten von Rundzellensarkomen Blutungen resp. den später daraus gebildeten nicht melanotischen Farbstoff gefunden hat.

Noch auffallender als die Verschiedenheit der Auffassung von Sarkoid und Sarkom in histologischer Beziehung ist die der anatomischen Veränderungen bei der Mycosis fungoides von Seiten der einzelnen Autoren.

Nach Neisser besteht der histologische Bau eines Knotens der Mycosis fungoides „aus einkernigen Zellen mit rundem Kern, die in ein zartes Bindegewebsreticulum eingelagert, die Cutis von der Ober-

1) Die Histopathol. der Hautkrankheiten. Berlin 1894. 
fläche an bis zum subcutanen Gewebe durchsetzen". Er nähert sich am meisten den infectiösen Granulationstumoren, zu denen er aber nicht gerechnet werden darf, weil sein specifischer Erreger noch nicht gefunden ist. Möglicherweise ist nach Neisser die Affection den Kundrat'schen Vegetationsstörungen zuzurechnen.

Ganz ähnlich drïckt sich Kaposi aus. Nach ihm bestehen die Knoten ebenfalls aus kleinen Rundzellen, die in ein Maschenwerk feinen fibrillären Bindegewebes eingelagert sind, während das autochthone Gewebe dabei nicht durch das Geschwulstgewebe substituirt wird.

Im Gegensatz zu diesen doch sehr allgemein gehaltenen Angaben fallen die ausserordentlich zahlreichen histologischen Specifitäten auf, die Unna bei Mycosis fungoides gefunden hat, besonders dem Rundzellensarkom gegenüber, wenngleich er zugiebt, „dass in den späteren Stadien des Sarkoms, wenn der plasmomatöse Charakter der Geschwulst zurücktritt, der Unterschied gegen die Mycosis fungoides sehr zurücktritt".

Da seine Angaben nicht in die Lehrbücher der soeben genannten Autoren aufgenommen sind, muss man allerdings wohl annehmen, dass sie deren Anerkennung nicht gefunden haben.

Bei der Zusammenstellung der beiderseitigen Merkmale heisst es bei Unna ungefähr:

Bei Sarkom giebt es nicht die bei Mycosis oft vorkommende rein flächenhafte Ausbreitung; die Zellen der Mycosis fungoides sind polymorph, die des Rundzellensarkoms uniform. - Die ersteren enthalten oft mehrere Kerne, auch wenn der Zellleib schon abgebröckelt ist.

Bei der Mycosis fungoides finden sich viel Lymphspalten und Lymphgefässe, die mit chromophilen Bröckeln beladen sind. Ferner giebt es bei derselben viel Mastzellen, die sich lange halten, während sie bei der Sarkomatosis bald zu Grunde gehen.

$\mathrm{Zu}$ bemerken ist hierbei, dass Unna keinen Unterschied der Befunde bei der gewöhnlich vorkommenden Form der Mycosis macht von denjenigen bei der Vidal'schen Gruppe.

Wenn nun auch in unserem Fall in den Lymphspalten das Granoplasma sich nicht ganz sicher nachweisen liess, bleiben nach dem Unnaschen Schema genügend viel histologische Charakteristica bestehen, um auf Grund derselben ihn als Mycosis fungoides bezeichnen zu können. -

Was nun die hier am meisten interessirenden Veränderungen der Thränendrïsen und Conjunctiven der Augen anbetrifft, so dürften dieselben bei der Mycosis fungoides noch nicht beschrieben sein. Besonders bemerkenswerth ist dabei, dass erst die Erkrankung der Augen die Patientin zum Arzt führte. 
Nach Groenouw ${ }^{1}$ ) sind einige Beobachtungen in der Literatur verzeichnet, denen zufolge Knoten unter der Lidhaut aufgetreten sind, was bei dem allgemeinen Sitz der Geschwulst unter der Cutis naturlich nicht auffallend ist.

Oefter sind dagegen Veränderungen an den Augen bei allgemeiner Leukämie und Pseudoleukämie beschrieben worden, wenn auch - so weit ich die Literatur durchsehen konnte - niemals bei den oben abgehandelten Formen, die sich durch hauptsächliche Localisation der Lymphome in der Cutis charakterisiren.

Die dabei beobachteten symmetrischen Tumoren der Orbita, der Exophthalmus, die Geschwülste in Bindehaut und Thränendrüse etc., geben den Beschreibungen und Abbildungen nach jedoch ein so grundverschiedenes Bild gegen unseren Fall, dass man fast daraus schon bei dem letzteren die fraglichen Blutkrankheiten als Ursache ausschliessen kann.

Klar gestellt werden die vorläufig so geheimnissvollen Krankheitsbilder der Mycosis fungoides, der Leucaemia und Pseudoleucaemia cutis, des Hautsarkoids etc. sicherlich erst werden, wenn noch mehr neue Methoden zur histologischen Forschung oder gar ätiologische Charakteristica gefunden sind. Wenn nun auch in dieser Beziehung unser Fall vorläufig nur von beschränktem Werthe ist, dürfte er bei der enormen Seltenheit hierher gehöriger Beobachtungen als Vervollständigung des Symptomenbildes etc. schon jetat eine gewisse Beachtung zu beanspruchen haben.

1) Graefe-Saemisch. 1901. Bd. XI. S. 234. 